\title{
Professional Service Firms
}

\author{
Stephan Kaiser und Georg Loscher
}

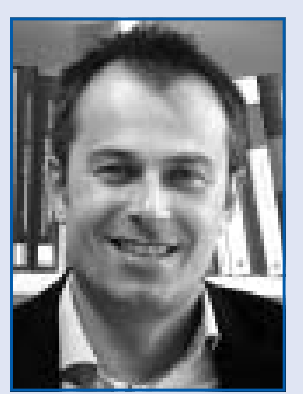

Prof. Dr. Stephan Kaiser ist Inhaber der Professur für Personalmanagement und Organisation an der Universität der Bundeswehr München, seine Forschungsschwerpunkte liegen im Bereich Führung von Professional Service Firms, organisationale Routinen und HumanressourcenManagement.

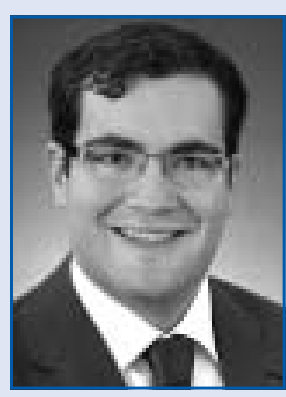

Dipl.-Kfm. Georg Loscher ist wissenschaftlicher Mitarbeiter an der Professur für Personalmanagement und Organisation an der Universität der Bundeswehr München, seine Forschungsinteressen umfassen Steuerung von Professional Service Firms und Praxistheorien.

Professional Service Firms als wissensintensive Dienstleistungsunternehmen unterscheiden sich durch ihre kritischen Ressourcen Wissen, Beziehungskompetenz und Reputation stark von herkömmlichen Unternehmen. Dies stellt spezielle Anforderungen an das Management von Professional Service Firms und an die Führung von Professionals als Träger der kritischen Ressourcen.

Stichwörter: Professional Service Firms, Dienstleistungen, immaterielle Ressourcen

\section{Begriff und Typisierung}

Die Bezeichnung „Professional Service Firms“ ist in der angelsächsischen Managementforschung seit langem etabliert und wird zunehmend auch in der deutschsprachigen Forschung und Praxis verwendet. Als Professional Service Firms (PSF) werden Unternehmen bezeichnet, die im Regelfall für andere Unternehmen wissensintensive Dienstleistungen erbringen. Diese wissensintensiven Dienstleistungen sind mannigfaltiger Natur, sie reichen von rechtlichen Dienstleistungen über Unternehmensberatung bis hin zu Marketingdienstleistungen. Alle haben jedoch die hohe Bedeutung von Mitarbeitern in der Leistungserstellung gemeinsam. Diese werden in PSF häufig als Professionals bezeichnet. Sie genießen aufgrund ihres einzigartigen Wissens eine sehr große Autonomie in der Leistungserbringung und besitzen dem Unternehmen gegenüber ein hohes Maß an Verhandlungsmacht (vgl. Bürger, 2005, S. 21 ff.; Kaiser/Ringlstetter, 2011, S. 3 ff.).

Ausgehend von den „Professionals“ werden von Nordenflycht (2010) zwei Arten von PSF unterschieden: Die klassischen berufsständisch organisierten PSF und die NeoPSF. Die klassischen PSF organisieren sich über Kammern, in denen die Professionals den Zugang zum Beruf über Berufsexamina, ihre Rechte und Pflichten sowie die Mindestqualität der Dienstleistung regeln (z. B. Steuerberatung, Rechtsanwälte und Wirtschaftsprüfer). Fehlt die „Verkammerung“ des Berufs und treffen aber alle anderen Merkmale wie Wissensintensität, Dienstleistungscharakter und Unternehmenskunden zu, spricht man von Neo-PSF (z. B. Unternehmensberatungen, Werbeagenturen, Ingenieurdienstleister, IT-Beratungen u.ä.; vgl. Nordenflycht, 2010, S. 165 ff.). PSF als Branche vereinigen somit unterschiedlichen Teilbranchen, die jedoch spezifische Gemeinsamkeiten aufweisen.

Im Folgenden wird daher der Frage nachgegangen, welche konstitutiven Merkmale PSF prägen, auf welche wettbewerbskritischen Ressourcen PSF zurückgreifen und welche Managementimplikationen sich hieraus ergeben.

\section{Konstitutive Merkmale von PSF}

Professional Service Firms weisen drei konstitutive Merkmale auf: Den Dienstleistungscharakter, die Unternehmensbezogenheit und die Professionalität bzw. Wissensintensität (vgl. ähnlich Kaiser/Ringlstetter, 2011, S. 3 ff.).

Mit dem Dienstleistungscharakter sind zwei Aspekte verknüpft. Zum einen ist dies die Immaterialität der Leistungen. Diese führt beim Kunden zu Unsicherheit, da er sich auf das Leistungsversprechen der PSF verlassen muss und die Qualität der Dienstleistung erst nach der Erbringung bzw. teilweise aufgrund eines fehlenden Vergleichsergebnisses überhaupt nicht nachprüfen kann. Man spricht in diesem Zusammenhang von ,opaker“ Qualität (vgl. Nordenflycht, 2010, S. 161).

Einen zweiten Aspekt bildet die Interaktion der Mitarbeiter der PSF mit Mitarbeitern der Kundenunternehmen. PSF können ihre Dienstleistungen nur erbringen, wenn sie den Kunden als externen Faktor in die Erstellung der Dienstleistung integrieren, d. h. mit ihm mehr oder weniger intensiv kommunizieren und interagieren.

Das Merkmal ,Unternehmensbezogenheit“" verweist darauf, dass die Kunden von PSF grundsätzlich Unternehmen sind. Dies erhöht die Komplexität der Leistungserbringung, da der Adressat nicht ein Individuum, sondern aus einer Vielzahl von Personen besteht. Gleichzeitig ha- 


\begin{tabular}{|l|l|}
\hline Konstitutive Merkmale & \\
\hline Dienstleistungscharakter & $\begin{array}{l}\text { Immaterialität von Leistungen } \\
\text { - Hoher Interaktionsgrad }\end{array}$ \\
\hline Unternehmensbezogenheit & $\begin{array}{l}\text { Komplexität der Leistungserbringung } \\
\text { Notwendigkeit von unternehmensspezifischen } \\
\text { Kenntnissen }\end{array}$ \\
\hline $\begin{array}{l}\text { Professionalität und } \\
\text { Wissensintensität }\end{array}$ & $\begin{array}{l}\text { Lösung komplexer und unstrukturierter Probleme } \\
\text { Wertschöpfung durch organisationale Wissensbasis }\end{array}$ \\
\hline
\end{tabular}

Tab. 1: Konstitutive Merkmale von PSF ben die Ergebnisse einen großen Einfluss auf die Kundenorganisation, was ein hohes Maß an unternehmensspezifischen Kenntnissen seitens der PSF erfordern kann.

Letzteres ist bereits mit dem Merkmal der Professionalität und Wissensintensität verknüpft. PSF lösen komplexe und unstrukturierte Probleme. Dies erfordert ein hohes $\mathrm{Ma}$ an organisationalem und individuellem Wissen als Basis der Problemlösungsfähigkeit, da die Wertschöpfung der PSF auf dem Einsatz ihrer organisationalen Wissensbasis beruht.

\section{Kritische Ressourcen}

PSF greifen auf drei miteinander in Beziehung stehende, wettbewerbskritische Ressourcen zurück, um am Markt langfristig erfolgreich zu agieren: professionelles Wissen, Beziehungskompetenz und Reputation.

Professionelles Wissen stellt die erste kritische Ressource von PSF zur Lösung komplexer Probleme dar. Dieses Wissen wird von Bürger (2005) in technisches Wissen und Kundenwissen unterschieden.

- Das technische Wissen umfasst die auf Fach- und Methodenwissen beruhenden Problemlösungsfähigkeiten der Professionals. Es stellt die generelle Voraussetzung zur Lösung des Kundenproblems dar.

- Kundenwissen bezeichnet die Kenntnisse über Branchen, Unternehmensspezifika und Entscheidungsträgern des Kundenunternehmens. Diese Wissensart bezeichnet die spezifische Voraussetzung zur Erstellung kundenspezifischer Problemlösungen.

Träger der kritischen Ressource Wissen sind einerseits die einzelnen Professionals, die ihr Wissen im Rahmen ihrer Ausbildung und im Laufe der Sozialisation innerhalb der PSF erwerben. Andererseits stellt die Organisation eine Wissensbasis bereit, die auf der Gesamtheit des Wissens und der Problemlösungsfähigkeiten der Organisationsmitglieder beruht (vgl. Tordoir, 1995, S. 20 ff.; Bürger, 2005, S. 38 ff.).

Die Beziehungskompetenz stellt nach Bürger (2005) die zweite kritische Ressource von PSF dar. Diese teilt sich auf in die auftragsspezifische und die auftragsübergreifende Beziehungskompetenz.

- Die auftragsspezifische Beziehungskompetenz bezeichnet die Fähigkeit zur Integration des Kunden während einer einzelnen Auftragsepisode. So kann zum einen auftragsspezifisches Wissen integriert werden, zum anderen kann neues branchen- oder unternehmensspezifisches Wissen generiert werden. Wesentlich für die Interaktion zwischen PSF und Kunden sind die Fähigkeiten der Professionals, eine verlässliche Arbeitsbeziehung aufzubauen, die dem Kunden als Qualitätssurrogat dienen kann.

- Die auftragsübergreifende Beziehungskompetenz beschreibt die Fähigkeit, über einzelne Auftragsepisoden hinaus Kontakt mit dem Kunden zu halten und eine langfristige Dienstleister-Kundenbindung aufzubauen. Diese dient als Ausgangspunkt zur Generierung weiterer Aufträge. Eine entscheidende Rolle spielen dabei die persönlichen Beziehungen zwischen Senior-Professionals und den Entscheidungsträgern der Kundenorganisation, aus deren Interaktion neue Möglichkeiten für Dienstleistungsaufträge entstehen.

Beide Arten der Beziehungskompetenz helfen den Kunden, durch die wahrgenommene Interaktionsqualität auf die opake Dienstleistungsqualität zu schließen und so Vertrauen in die Problemlösungsfähigkeit der PSF aufzubauen (vgl. Bürger, 2005, S. 43 ff.; Lфwendahl, 2005, S. 88 f.).

Die dritte kritische Ressource von PSF stellt ihre Reputation dar. Dienstleistungen von PSF stellen aufgrund ihrer opaken Qualität in hohem Maße Vertrauensgüter dar. Um das Risiko eines Fehlkaufs einer komplexen Dienstleistung zu minimieren, nutzen die Kunden die Reputation einer PSF zur Validierung ihrer eigenen Entscheidung. Reputation kann daher als indirekter Ausdruck der Dienstleistungsqualität verstanden werden, denn sie stellt das Ergebnis der langfristigen Beziehungskompetenz und des erfolgreichen Einsatzes von Wissen in spezifischen Auftragssituationen bei unterschiedlichen Kunden dar. Zusätzlich dient die Reputation der PSF während der Auftragsbearbeitung dazu, das Risiko eines kundenschädlichen Verhaltens zu minimieren, da die PSF durch Enttäuschung des Qualitätsversprechens ihre Reputation und damit zukünftige Aufträge schnell verlieren kann (vgl. Scott, 2001, S. XII ff.; Greenwood/Empson, 2003, S. 913 ff.).

\section{Managementimplikationen}

Die Immaterialität wissensintensiver Dienstleistungen hat für das Management einer PSF wichtige Implikationen. So ist die Qualität der Dienstleistungserbringung durch den 
Kunden nur schwer einschätzbar. Dennoch entscheidet die Qualitätswahrnehmung langfristig über den Erfolg der PSF. Daher beeinflussen gelungene Interaktionsepisoden zwischen Professionals und Kunden als Ersatzkriterium die Wahrnehmung der Qualität. Das Gelingen von Interaktionen wird dabei direkt durch die Beziehungskompetenz und Motivation des Professionals sowie indirekt durch die organisationale Wissensbasis zur Problemlösung beeinflusst.

Da Professionals Träger des Wissens sind und ihre Beziehungskompetenz herausragende Bedeutung in der Kundeninteraktion hat, müssen sie gebunden und motiviert werden. Professionals haben vier wichtige Bedürfnisse:

1. Aufgaben, die herausfordern und zur eigenen Entwicklung beitragen,

2. Autonomie in der Aufgabenerfüllung,

3. Mitbestimmung bei Entscheidungen in Projekten und

4. Anerkennung durch gleichrangige Professionals.

Diese Bedürfnisse resultieren einerseits aus der hohen Identifikation mit dem Beruf und seinen Aufgaben, andererseits aus dem Wunsch Lösungen für Kundenprobleme $\mathrm{zu}$ generieren. Um die hohe Eigenmotivation aufrecht $\mathrm{zu}$ erhalten, müssen PSF neben monetären Anreizen wie der Möglichkeit, Mit-Eigentümer zu werden, auch nicht-monetäre Anreize wie Entwicklungsmöglichkeiten, Fortbildungen sowie herausfordernde Projekte setzen. Die Motivation ist umso wichtiger, da die Güte des Einsatzes der kritischen Ressourcen von den Professionals abhängt (vgl. Hall, 1968, S. 93; Maister, 2007, S. 168 ff.).

Eine wesentliche Bedeutung für den Erfolg von PSF stellt ihre organisationale Wissensbasis dar, daher besteht eine Kernherausforderung für PSF im Management des vorhandenen Wissens. Dazu können nach Hansen (1999) zwei Strategien unterschieden werden:

- Kodifikation: Ziel ist es, das Wissen der einzelnen Professionals oder neu generiertes Wissen über Datenbanken für alle Professionals innerhalb der PSF verfügbar zu machen. Der Leitgedanke dieser Strategie ist eine Art organisationales „Wissenslager“, in dem Wissen von den Professionals auf Dokumente übertragen wird. Die Kodifikation bezeichnet einen Standardisierungsprozess, durch den explizites Wissen durch alle Organisationsmitglieder genutzt werden kann. Daraus entsteht das Potential, Wissen in hohem Maße unabhängig von einzelnen Professionals nutzen zu können.

- Personalisierung: Diese Strategie zielt darauf ab, implizites und explizites Wissen einzelner Professionals von Person-zu-Person zu übertragen. Datenbanken dienen dabei als Unterstützung beim Finden passender Dialogpartner. Ziel der Strategie ist eine Individualisierung des Wissens und ein problem- und erfahrungsbasierter Austausch zwischen Professionals. Diese Strategie weist das Potential für Innovation und neue Problemlösungen auf, führt aber nur zu einer begrenzten Wiederverwertbarkeit von Wissen.
Die beiden Strategien treten oft in Mischformen auf, sie sind aber primär von der Art der Problemstellungen der PSF abhängig. Bei wiederkehrenden Problemstellungen bietet sich die Kodifikationsstrategie an, bei hoch-individualisierten Problemstellungen die Personalisierungsstrategie (vgl. Hansen, 1999, S. 106 ff.). Je nachdem wie die organisationale Wissensbasis eingesetzt wird, lassen sich unterschiedliche strategische Weiterentwicklungen der PSF abgrenzen. So kann das Wissen um Unternehmensspezifika der Kunden genutzt werden, in dem neue Projekte beim gleichen Kunden generiert werden (Client Leverage). Oder aber das bereits vorhandene Wissen wird auf Probleme neuer Kunden angewendet (Knowledge Leverage; vgl. Ringlstetter/Bürger, 2004, S. 286 f.).

Aus dem Zusammenspiel hochmotivierter Professionals und passendem Wissen für die Problemlösung entstehen gelungene Interaktionsepisoden, die zur Zufriedenheit des Kunden mit der sonst schwierig beurteilbaren Dienstleistungsqualität führen. Aus den einzelnen Aufträgen wächst eine Vertrauensbeziehung zwischen dem Kundenunternehmen und der PSF, die zu weiteren Aufträgen führt. Indirekt speist sich aus diesem Vertrauensverhältnis zwischen Kunden und PSF auch die Reputation, denn andere Kunden erwarten die Übertragung von gelungenen Dienstleistungsbeziehungen auf ihre eigenen Aufträge.

\section{Fazit}

PSF als wissensintensive Dienstleistungsunternehmen sind essenziell auf ihre Mitarbeiter angewiesen. Diese tragen mit ihrer Beziehungskompetenz und ihrem Wissen wesentlich zu einer hohen Dienstleistungsqualität und damit langfristig zur Reputation der PSF bei. Die Führung von Professionals und das Management der kritischen Ressourcen stellt eine hohe Herausforderung an die PSF. Obgleich die Kunden auf PSF mit ihrem Schatz an Problemlösungsfähigkeiten und Kenntnissen als Begleiter in Entscheidungen angewiesen bleiben, steigen die Anforderungen an die Professionals und ihr Wissen weiter, so dass ein bewusster Einsatz der kritischen Ressourcen für PSF immer wichtiger wird.

\section{Literatur}

Bürger, B., Aspekte der Führung und der strategischen Entwicklung von Professional Service Firms: Der Leverage von Ressourcen als Ausgangspunkt einer differenzierten Betrachtung, Wiesbaden 2005.

Greenwood, R., L. Empson, The professional partnership: Relic or exemplary form of governance?, in: Organization Studies, 24. Jg. (2003), S. 909-933.

Hall, R. H., Professionalization and bureaucratization, in: American Sociological Review, 33. Jg. (1968), S. 92-104.

Hansen, M. T., The search-transfer problem: The role of weak ties in sharing knowledge across organization subunits, in: Administrative Science Quarterly, 44. Jg. (1999), S. 82-111.

Kaiser, S., M. Ringlstetter, Strategic Management of Professional Service Firms: Theory and Practice, Heidelberg et al. 2011.

Løwendahl, B., Strategic management of professional service firms, Kopenhagen 2005. 
Maister, D. H., Managing the professional service firm, London 2007.

Nordenflycht, A. von, What Is a Professional Service Firm? Toward a Theory and Taxonomy of Knowledge-Intensive Firms, in: Academy of management review, 35. Jg. (2010), S. 155174.

Ringlstetter, M., B. Bürger, Strategische Entwicklung von Professional Service Firms - Optionen, Herausforderungen und Um- setzungsformen, in: S. Kaiser, B. Buerger, M. Ringlstetter (Hrsg.), Strategien und Management für Professional Service Firms, Weinheim 2004, S. 283-305.

Scott, M., The Professional Service Firm: A Manager's Guide to Maximising Profit and Value, Chichester 2001.

Tordoir, P. P., The professional knowledge economy, Dordrecht 1995.

\section{Vorschau auf WiSt Heft 2/2015}

- Prof. Dr. Thomas Wrona und Corinna Sinzig, M.Sc., Nichtmarktstrategien - Begriffsbestimmung, Abgrenzung, Determinanten

- M. Sc. Dominik Schneller, Scheinregressionen in Zeitreihendaten

- Dipl.-Kfm. Jacob Kleinow, MMMag. Christof Morscher und Univ.-Prof. Dr. Andreas Horsch, Systemrelevante Finanzinstitute

- Prof. Dr. Alfred Endres, Das Erneuerbare-Energien-Gesetz (EEG) und seine Reform - Grundsätzliche Überlegungen aus ökonomischer Sicht

- Dr. Sabine Spangenberg, The Euro: A German Tale

- Dr. Christian Kreiß, Geplanter Verschleiß

- Prof. Dr. Gunther Meeh-Bunse und Dipl. Wirtschaftsjuristin Anke Hermeling, Änderung der EU-Rating-Verordnung

- Prof. Dr. Gerhard Feldmeier und Prof. Dr.-Ing. Peter Ritzenhoff, "Studienführerschein“ als Leitplanke für ein erfolgreiches Hochschulstudium

- M.Sc. Debbie Claassen, Prof. Dr. Christian Hofmann und B.Sc. Nina Kühne, Kostentreiberplanung am Beispiel der Deutschen Lufthansa AG - Teil 2: Lösungen 